\section{0-028 OPERCULAR INDEX SCORE (OIS): A NOVEL PREDICTOR OF COLLATERAL ROBUSTNESS AND NEUROLOGIC OUTCOMES IN THE ENDOVASCULAR MANAGEMENT OF ACUTE ISCHEMIC STROKE}

${ }^{1} \mathrm{~A}$ Copelan, ${ }^{1} \mathrm{M}$ Chehab, ${ }^{1} \mathrm{~S} X \mathrm{Xli},{ }^{2} \mathrm{~W}$ Brinjikji, ${ }^{3} \mathrm{Z}$ Wilseck, ${ }^{2} \mathrm{D}$ Kallmes, ${ }^{1} \mathrm{~J}$ Wilseck. ${ }^{1}$ Radiology, Oakland University William Beaumont School of Medicine, Royal Oak, MI ${ }^{2}$ Radiology, Mayo Clinic, Rochester, MN; ${ }^{3}$ Radiology, University of Michigan, Ann Arbor, MI

\subsection{6/neurintsurg-2016-012589.28}

Purpose Assessment of collaterals is imperative in patient selection for endovascular reperfusion in acute ischemic stroke (AIS). The purpose of this study was to evaluate the correlation between a novel CTA based collateral scoring system: the Opercular IndexScore (OIS), with the capillary index score (CIS) at angiography and neurologic outcomes at 90 days following endovascular treatment of acute ischemic stroke.

Materials and methods Data from 58 patients with AIS who underwent clinical assessment, institutional stroke based CT imaging (noncontrast CT, CT Perfusion with CTA reconstructions) and endovascular reperfusion were included in this study. OIS was retrospectively calculated from CTA images as the ratio of the number of opacified branches in the Sylvian fissure on the normal side to those on the affected side and dichotomized into favorable OIS (fOIS i.e. OIS $\leq 2$ ) and poor OIS (pOIS i.e. OIS $>2$ ). CIS was defined as favorable $(\mathrm{CIS} \geq 2$ ) or unfavorable (CIS $<2$ ). Good neurological outcome was defined as $\mathrm{mRS} \leq 2$ at 90 days. Baseline clinical, CT and angiographic variables between fOIS and pOIS were compared using students t-test for continuous and chi-squared test for categorical variables. Multivariate regression analysis was utilized to identify correlation of any variables with a good neurologic outcome. The ability of fOIS to predict a good neurologic outcome was assessed using sensitivity, specificity, positive predictive value, negative predictive value and AUC using an ROC analysis.

Results Thirty-five patients had fOIS and 20 patients had pOIS. There was no difference in mean age $(p=0.96)$, gender $(p=0.31)$, side of occlusion $(p=0.11)$, use of IV-tPA $(p=0.73)$, ASPECTS $(p=0.61)$, door-to-puncture time $(p=0.49)$, door to recanalization time $(p=0.81)$, recanalization rate $(0.32)$ or TICI scores $(p=0.78)$. There was a trend towards lower NIHSS in the fOIS group ( $\mathrm{p}=0.07$ ). At angiography, $82.9 \%$ of patients ( $n=29$ ) with fOIS had a fCIS and $40.0 \%$ of patients $(n=14)$ with pOIS group had a fCIS $(\mathrm{p}=0.002)$. Patients with fOIS had an $80.0 \%(\mathrm{n}=28)$ rate of good neurological outcomes compared to $15.0 \%(\mathrm{n}=3)$ in the pOIS group ( $<<0.0001)$. On multivariate logistic regression analysis adjusting for baseline NIHSS, OIS and device used, a favorable OIS was the only variable independently associated with good neurological outcome $(\mathrm{OR}=17.2,95 \%$ $\mathrm{CI}=3.8-104.3)$. In predicting good neurological outcome, fOIS demonstrated a sensitivity of $90.3 \%$, specificity of $70.8 \%$, positive predictive value of $80.0 \%$ and negative predictive value of $85 \%$. The AUC was 82.2. Interobserver agreement was substantial with a kappa value of $0.6495 \% \mathrm{CI}=0.41-0.88$.

Conclusion OIS is a practical, noninvasive scoring system that can be used to predict collateral robustness and good clinical outcome among patients undergoing endovascular recanalization in the treatment of acute ischemic stroke.

Disclosures A. Copelan: None. M. Chehab: None. S. Xli: None. W. Brinjikji: None. Z. Wilseck: None. D. Kallmes: None. J. Wilseck: None.

\section{$0-029$ ACUTE THROMBUS FORMATION ON FLOW DIVERTERS IMAGED IN VIVO USING OPTICAL COHERENCE TOMOGRAPHY}

${ }^{1} \mathrm{M}$ Marosfoi, 'E Langan, 'S Vedantham, ${ }^{1} \mathrm{~F}$ Clarençon, ${ }^{1} \mathrm{R}$ King, ${ }^{2} \mathrm{~J}$ Wainwright, ${ }^{1} \mathrm{M}$ Gounis, ${ }^{1}$ A Puri. ${ }^{1}$ Radiology, University of Massachusetts, Worcester, MA; ${ }^{2}$ Research, Medtronic Neurovascular, Invine, CA

\subsection{6/neurintsurg-2016-012589.29}

Introduction In vitro studies have shown that Pipeline Embolization Device+Shield Technology (Shield) with a surface modification of a $3 \mathrm{~nm}$ thick modified phosphorylcholine is less thrombogenic. ${ }^{1}$ We hypothesize that Shield has less thrombus formation in vivo as compared to Pipeline Embolization Devices (PED) regardless of dual antiplatelet therapy (DAPT).

Methods Forty rabbits with elastase induced aneurysms were randomly assigned to receive a Shield or PED. For each device, half of the animals received DAPT. In each of the four groups, 10 animals were enrolled for a period of 30 days. Herein, we report on 32 animals that have reached the study endpoint to date. Animals that received DAPT (10 mg/ $\mathrm{kg}$ each of aspirin and clopidogrel) were started a 5 days prior to implant and continued until the endpoint at 30 days. At the time of implant optical coherence tomography (OCT, Dragonfly, St Jude) was performed before and after angioplasty, and repeated at terminal follow-up. Thrombus formation was assessed at 4 locations along the implant (distal end, at the level of the vertebral artery, at the aneurysm neck, and at the proximal end) as present or absent. Aneurysm occlusion was assessed on digital subtraction angiography after 30 days and according to the scale of Darsaut et al. ${ }^{2}$

Results Baseline characteristics (e.g., aneurysm size, neck size, parent vessel diameter) were not different between the four groups $(p>0.1)$. Animals receiving DAPT had a significant reduction in PRU values $(69 \pm 28$ vs $247 \pm 41$, $\mathrm{p}<0.0001)$ and no change in ARU $(649 \pm 31$ vs $659 \pm 9$, $\mathrm{p}>0.05)$. The Shield was more likely to have no thrombus

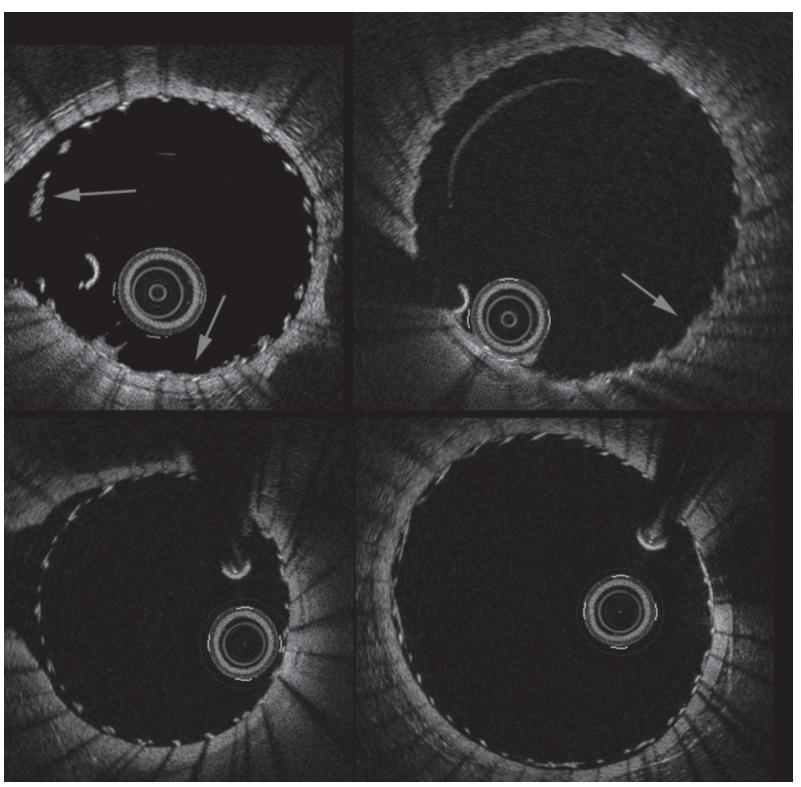

Abstract 0-029 Figure 1 Top panel shows thrombus formation on the PED surface (arrows) absent in the Shield device (bottom panel), after implantation. Left images taken at the origin of the vertebral artery, and right images are acquired proximal to the aneurysm 